\title{
THE SHAPE-STRUCTURE RELATION FOR THE LIGHT CONSTRUCTION MEMBRANE TYPE
}

\author{
L. Kopenetz ${ }^{\text {a }}$, A. Cătărig a , Mihaela-Teodora Ghemiş ${ }^{\text {b, * }}$ \\ a Technical University of Cluj-Napoca, Faculty of Civil Engineering, Mechanics of Structures Department, Romania \\ ${ }^{\mathrm{b}}$ University of Oradea, Faculty of Civil Engineering, Cadastre and Architecture, Civil Engineering Department, Romania
}

Received: 04.09.2015 / Accepted: 01.10.2015 / Revised: 17.11.2015 / Available online: 15.12.2015

DOI: $10.1515 /$ jaes-2015-0020

KEY WORDS: membrane, light structures, structure's shape, finite elements, soap film

\begin{abstract}
:
In the case of light structures membrane type the form is confused with the structure and vice versa. Thus the analysis process, nonlinear type, the one for form finding is also a means of optimizing these structures. To respect the natural principle of minimum it is advisable that the structure's shape is similar to the minimum surface area. The numerical problem solving is based on using finite elements with constant strain of soap film. Based on these considerations, the paper presents aspects of determining the shape of the membrane structure using finite elements of soap film.
\end{abstract}

\section{INTRODUCTION}

Light structures membrane type or membrane with cables allows the most varied and complex monumental engineering construction (Kopenetz \& Cătărig, 2006; Kopenetz \& Pârv, 2014; Kopenetz, \& Prada, 2011).

Examples of such structures are shown in the following:

a) Juventus Stadium, Turin, Italy (figure 1) - Structural solution characterized by a roof suspended from steel cables connected by two pillars and the main structural system (consisting of reinforced concrete beams to level $+18.55 \mathrm{~m}$ and steel beams up to the external supports of the roof, elevation $+33.00 \mathrm{~m}$ ).

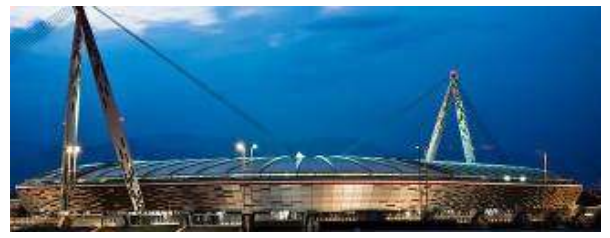

Figure 1. Juventus Stadium, Turin, Italy (http://www.italia.it/uploads/RTEmagicC_Torino_Juventus_Stadi um_www.juventus.com.jpg.jpg) (view at 18 Aug. 2015)

b) Florida Suncoast Dome (Tropicana Field), Saint Petersburg, Florida, USA (figure 2) - "Tensegrity" structure with radial cable systems. The canvas roof is made of fibreglass coated with Teflon.

\footnotetext{
* Corresponding author: e-mail: mihaelatoadere@yahoo.com
} 


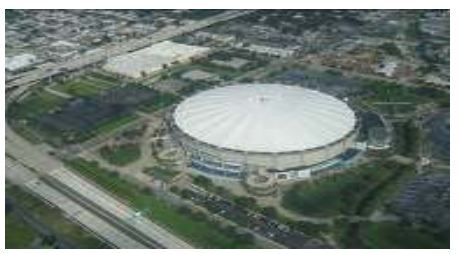

Figure 2. Florida Suncoast Dome (Tropicana Field), Saint Petersburg, Florida, USA

(https://upload.wikimedia.org/wikipedia/commons/0/09/Tropicana field_from_air.JPG) (view at 18 Aug. 2015)

c) Tao-Yuan County Arena, Taoyuan, Taiwan (figure 3) - Membrane Structure having a circular plan with $120 \mathrm{~m}$ in diameter. The membrane made of PTFE (polytetrafluoroethylene) having a long life.

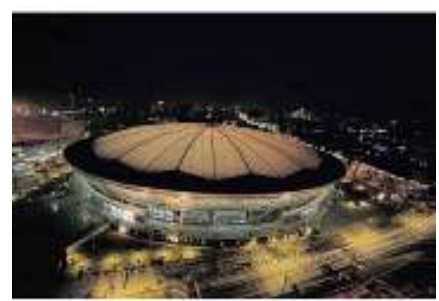

Figure 3. Tao-Yuan County Arena, Taoyuan, Taiwan (http://www.taiyokogyo.co.jp/img/lgr/mk_371.jpg) (view at 18 Aug. 2015)

d) Hajj Terminal, Jeddah, Saudi Arabia (figure 4) Structure made of Teflon membrane, covering an area of 105 hectares.

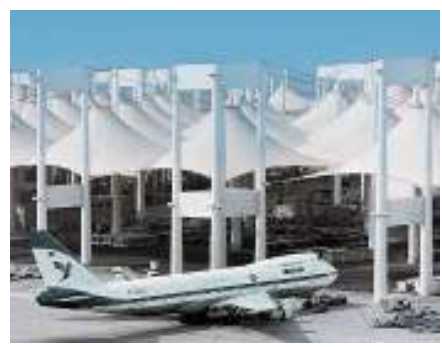

Figure 4. Hajj Terminal, Jeddah, Saudi Arabia (http://archrecord.construction.com/features/aiaAwards/10_25year Award/1.jpg ) (view at 18 Aug. 2015)

Given the flexibility of these structures in general, and structural subassemblies, in particular, the structural design must address two key issues:

- Determining the initial geometry (Kopenetz et al., 2004, 2005; (Kopenetz, 2006).

- Establishing the tensions and surface shape, from pre-stress and static and dynamic loads applied (Kopenetz \& Ionescu, 1985).
If for determining the stresses there are relatively many bibliographic alerts, for the form study there are few communications (Bentley, 1999; Woodbury, 2010).

To respect the natural principle of minimum it is advisable that the light structure's shape is similar to the minimum area surface.

The analytical solving of the minimal surface shape is possible only for certain specific contours (coil, askew quadrangle with tips on a regular tetrahedron etc.) (Fox \& Kemp, 2009; Burry, 2011).

From the point of view of structural engineering resolutions are based on PLATEAU's problem (Plateau, 1873), that means finding a minimal area surface for a closed space contour (Kopenetz \& Cătărig, 2006; Kopenetz \& Pârv, 2014).

\section{DETERMINATION OF MINIMUM AREA SURFACE}

Minimum area surfaces are those surfaces which of all the surfaces they pass through a skew curve, have the smallest area.

If on a closed elastic or rigid contour is considered a soap film with its own negligible weight, due to superficial stress, characteristic to fluid films, these take the form of minimum area surface, corresponding to the contours. In this regard, the minimum area surfaces represent the natural form of existence of films made of soap film, unloaded (Lynn, 1999; Hawking, \& Mlodinov, 2010).

The minimum area surface is determined in two conditions:

a) The first condition is related to the mean curvature:

$$
H=\frac{1}{2}\left(\frac{1}{R_{1}}+\frac{1}{R_{2}}\right)
$$

This curvature must be equal to zero in any point of the surface, which is equivalent to the condition $R_{1}=-R_{2}$, where $R_{1}$ and $R_{2}$ are the main radii of the curvature.

b) The second condition is related to stress, meaning that they must be constant at any point and in after each direction.

The approach of this condition, regarding the size of the minimum area surface, is experimental and 
numerical and it is followed by analytical methods only for particular cases.

The experimental determination of minimum area surface shape sums up to finding the coordinates and curvatures in all the points of the area, through stereophotogrammetry methods or even normal shooting from different angles. The numerical study, both for finding minimum area surface and for determining the shape from different loads, appeals to the special finite element with uniform stress $\sigma^{\mathrm{t}}$.

By discrete modelling of the fluid film surface, the membrane structure with an infinite number of degrees of freedom is replaced with a system with a finite number of degrees of freedom. This system represents a set of bidimensional membrane type elements, using isoparametric finite elements of indefinite shape, linked in knots (Kwinter, 2007; Rubinstein \& Firstenberg, 1999).

The finite elements used are Zienkiewicz-Irons type, the geometry and the allowed distribution of the displacement being presented using the same functions of the form and interpolation.

The implemented method of calculation is based on the theory presented in references (Kopenetz \& Ionescu, 1985; Kopenetz, et al., 2005,Kopenetz \& Cătărig, 2005; Kopenetz \& Pârv, 2014; Kopenetz \& Prada, 2011), considering only the linear-elastic response of the material.

Newton-Raphson iterative method is used in order to solve the stability issues which are not influenced by the type of the finite element. The differential equations of movement are solved using the Newark and Wilson methods.

The initial shape is set by the software in case of membrane type structures for which the initial shape is very important. The software uses a finite element having a constant tension as a pattern for the material of the surface structure. If there are no external loads, the minimum area is assumed as initial shape.

SUM01 calculation program developed to solve this problem is using efficiently an iterative process (Kopenetz \& Ionescu, 1985; Kopenetz \& Cătărig, 2006; Kopenetz \& Pârv, 2014; Kopenetz \& Prada, 2011).

\section{NUMERICAL EXEMPLES}

a) In order to determine the surface shape of a minimum area for the membrane type structure in figure 5 using two variants, let's consider point 12 fixed. The minimum surface area is calculated considering first the constant stress in the membrane equals $5 \mathrm{daN} / \mathrm{mm}$, and then $20 \mathrm{daN} / \mathrm{mm}$.

After analysing the results (table 1), we notice that as expected identical ordinates are obtained.

If point 12 is free, the minimum area is obtained as the horizontal plan, again an expected physical result.

\section{STARTING AREAS}
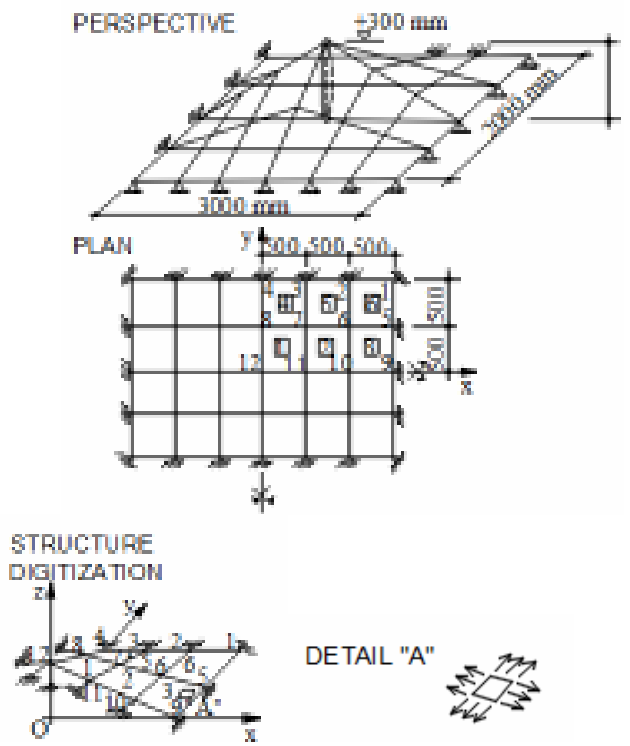

Figure 5. Shape of a minimum area for membrane structure

Material: Soap Film

\begin{tabular}{|c|c|c|c|c|c|c|}
\hline \multirow{3}{*}{ Point } & \multicolumn{5}{|c|}{ Coordinates } \\
\cline { 2 - 7 } & \multicolumn{3}{|c|}{ Starting } & \multicolumn{2}{c|}{$\begin{array}{c}\text { After } 10 \text { iterations with } \mathrm{t}_{0}=5 \\
\text { and } \mathrm{t}_{0}=20 \mathrm{daN} / \mathrm{mm}\end{array}$} \\
\cline { 2 - 7 } & $\mathrm{X}$ & $\mathrm{Y}$ & $\mathrm{Z}$ & $\mathrm{x}$ & $\mathrm{y}$ & $\mathrm{z}$ \\
\hline 1 & 1500 & 1000 & 0 & 1500 & 1000 & 0 \\
\hline 2 & 1000 & 1000 & 0 & 1000 & 1000 & 0 \\
\hline 3 & 500 & 1000 & 0 & 500 & 1000 & 0 \\
\hline 4 & 0 & 1000 & 0 & 0 & 1000 & 0 \\
\hline 5 & 1500 & 500 & 0 & 1500 & 500 & 0 \\
\hline 6 & 1000 & 500 & 50 & 985,56 & 498 & 20,66 \\
\hline 7 & 500 & 500 & 100 & 486,78 & 490,45 & 57,20 \\
\hline 8 & 0 & 500 & 150 & 0 & 437,33 & 75,44 \\
\hline 9 & 1500 & 0 & 0 & 1500 & 0 & 0 \\
\hline 10 & 1000 & 0 & 100 & 975,92 & 0 & 29,38 \\
\hline 11 & 500 & 0 & 200 & 422,2 & 0 & 84,55 \\
\hline 12 & 0 & 0 & 300 & 0 & 0 & 300 \\
\hline
\end{tabular}

Table 1. Coordinates $\mathrm{x}, \mathrm{y}, \mathrm{z}$ after 10 iterations 
b) In this example, the study of form is proposed for a bearing structure with pre-stressed membranes with arches, suitable for gyms (figure 6).
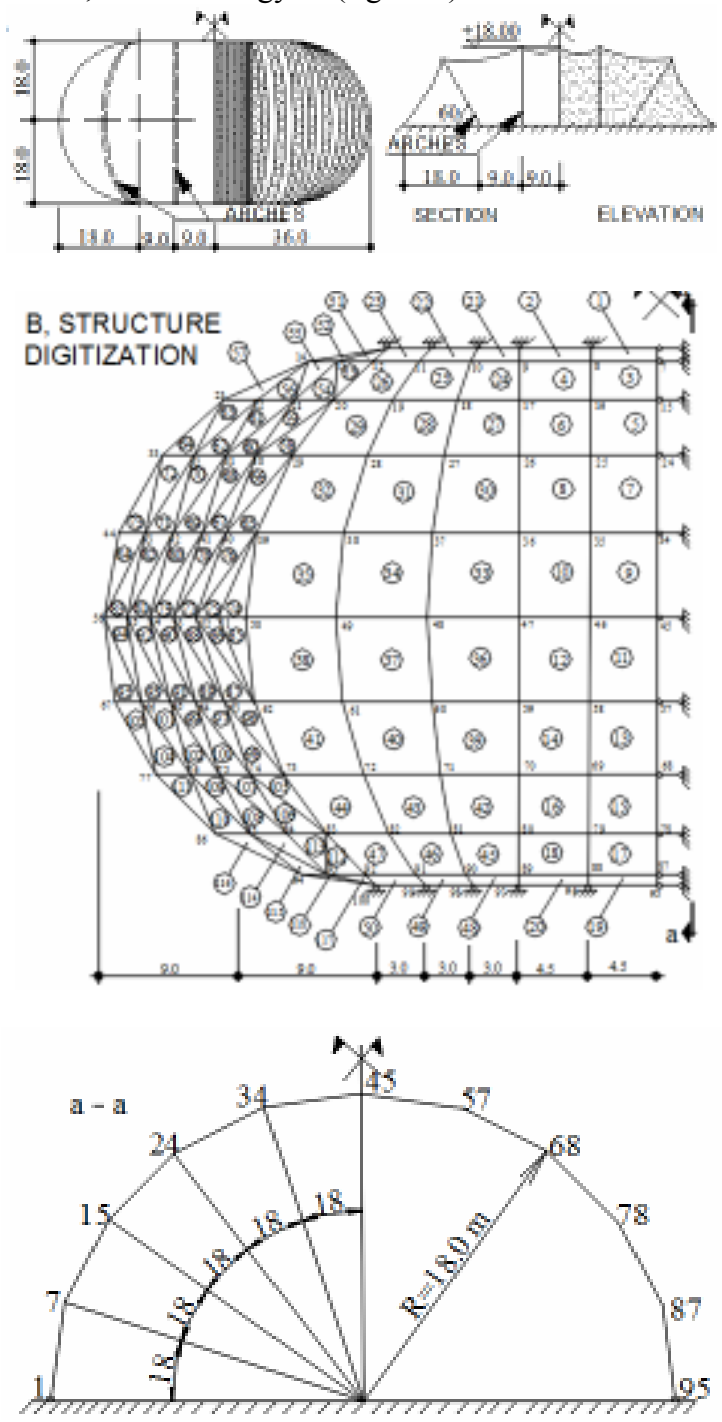

Figure 6. Prestressed membranes with arches suitable for gyms

The results are presented in figure 7 and table 2 :
LINE 33-29-26-24

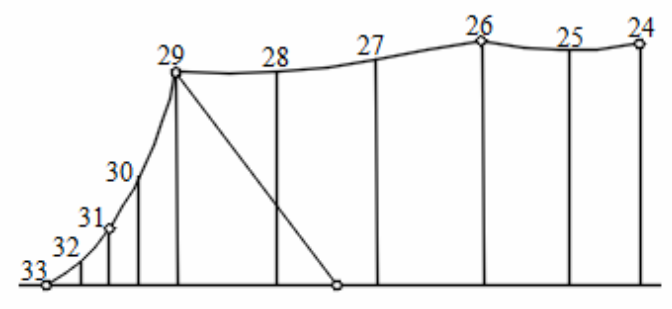

LINE 56-50-47-45
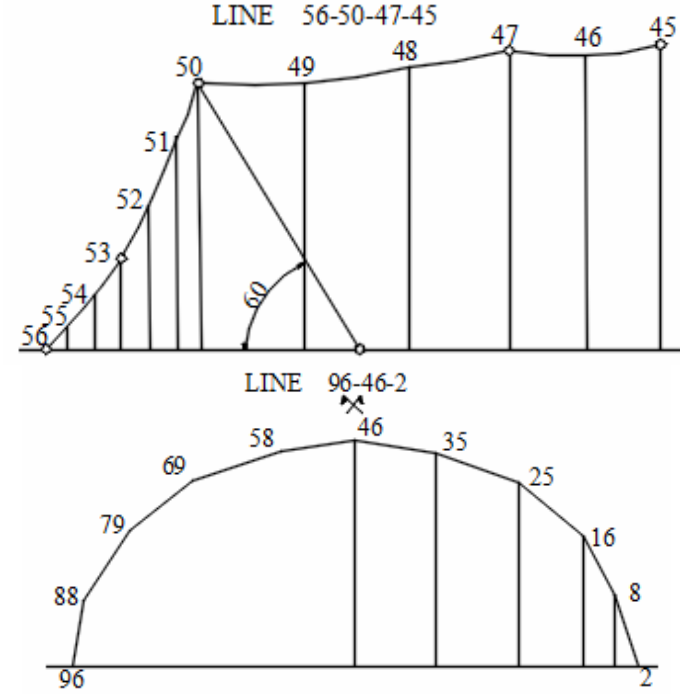

LINE $98-48-4$
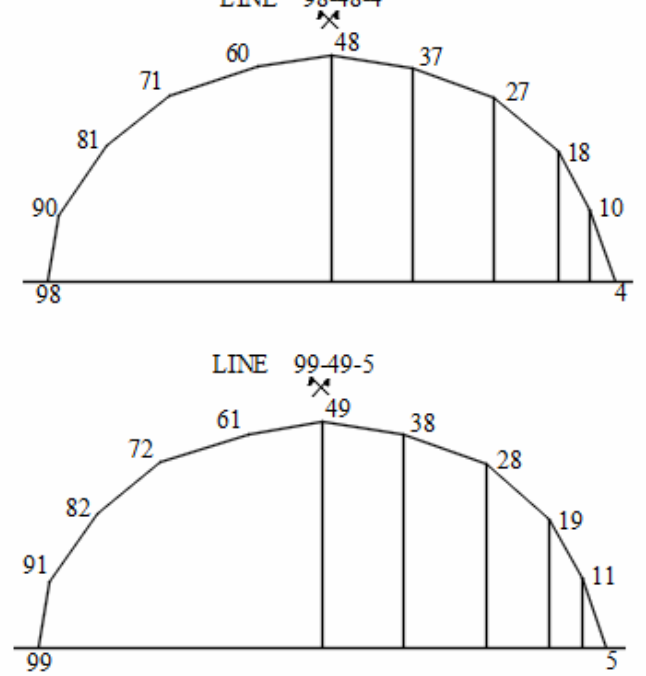

Figure 7. Shape result for prestressed membrane with arches suitable for gyms

Material: Soap Film

\begin{tabular}{|c|c|c|c|}
\hline \multirow{2}{*}{ Point } & \multicolumn{3}{|c|}{ Coordinates after 5 iterations [m] } \\
\cline { 2 - 4 } & $\mathrm{x}$ & $\mathrm{y}$ & $\mathrm{Z}$ \\
\hline 1. & 36,000 & 18,000 & 0 \\
\hline 2. & 31,500 & 18,000 & 0 \\
\hline 3. & 27,000 & 18,000 & 0 \\
\hline 4. & 24,000 & 18,000 & 0 \\
\hline 5. & 21,000 & 18,000 & 0 \\
\hline
\end{tabular}




\begin{tabular}{|c|c|c|c|}
\hline 6. & 18,000 & 18,000 & 0 \\
\hline 7. & 36,000 & 17,120 & 5,560 \\
\hline 8. & 33,115 & 16,820 & 5,513 \\
\hline 9. & 30,292 & 17,120 & 5,560 \\
\hline 10. & 27,423 & 16,618 & 5,203 \\
\hline 11. & 24,669 & 16,696 & 4,939 \\
\hline 12. & 22,077 & 17,120 & 4,830 \\
\hline 13. & 17,351 & 16,279 & 1,830 \\
\hline 14. & 12,440 & 17,120 & 0 \\
\hline 15. & 36,000 & 14,560 & 10,580 \\
\hline 16. & 34,110 & 14,447 & 10,483 \\
\hline 17. & 32,292 & 14,560 & 10,580 \\
\hline 18. & 29,714 & 14,134 & 9,820 \\
\hline 19. & 27,428 & 14,203 & 9,332 \\
\hline 20. & 25,495 & 14,560 & 9,160 \\
\hline 21. & 19,552 & 13,390 & 4,877 \\
\hline 22. & 13,472 & 13,628 & 1,965 \\
\hline 23. & 7,420 & 14,560 & 0 \\
\hline 24. & 36,000 & 10,580 & 14,560 \\
\hline 25. & 34,678 & 10,535 & 14,488 \\
\hline 26. & 33,412 & 10,580 & 14,560 \\
\hline 27. & 31,072 & 10,267 & 13,510 \\
\hline 28. & 29,135 & 10,352 & 12,890 \\
\hline 29. & 27,495 & 10,580 & 12,610 \\
\hline 30. & 22,019 & 9,798 & 8,091 \\
\hline 31. & 16,000 & 9,541 & 4,394 \\
\hline 32. & 9,747 & 9,874 & 1,801 \\
\hline 33. & 3,440 & 10,580 & 0 \\
\hline 34. & 36,000 & 5,560 & 17,120 \\
\hline 35. & 34,955 & 5,543 & 17,062 \\
\hline 36. & 33,955 & 5,560 & 17,120 \\
\hline 37. & 31,753 & 5,393 & 15,879 \\
\hline 38. & 30,027 & 5,447 & 15,195 \\
\hline 39. & 28,660 & 5,560 & 14,830 \\
\hline 40. & 23,756 & 5,220 & 10,551 \\
\hline 41. & 18,436 & 5,012 & 6,817 \\
\hline 42. & 12,760 & 5,007 & 3,837 \\
\hline 43. & 6,860 & 5,199 & 1,581 \\
\hline 44. & 0,880 & 5,560 & 0 \\
\hline 45. & 36,000 & 0 & 18,000 \\
\hline 46. & 35,031 & 0,001 & 17,946 \\
\hline 47. & 34,103 & 0 & 18,000 \\
\hline 48. & 31,933 & 0,002 & 16,690 \\
\hline 49. & 30,247 & 0,001 & 15,981 \\
\hline 50. & 28,850 & 0 & 15,580 \\
\hline 51. & 24,734 & $-0,003$ & 11,939 \\
\hline 52. & 20,352 & $-0,006$ & 8,460 \\
\hline 53. & 15,576 & $-0,005$ & 5,495 \\
\hline 54. & 10,511 & 0,010 & 3,105 \\
\hline 55. & 5,278 & 0,007 & 1,306 \\
\hline 56. & 0 & 0 & 0 \\
\hline
\end{tabular}

Table 2. Coordinates $\mathrm{x}, \mathrm{y}, \mathrm{z}$, after 5 iterations

c) The next example presents the study of the shape for a circular hall with membrane structure in three versions:

- Mechanical stress.

- Mneumatic stress.

- Pneumatic stress for cable supported structures.

The results are presented in figure 8, table 3, figure 9, table 4 , figure 10 and table 5 .
CIRCULAR WAREHOUSE

STUDY OF FORM FROM MECHANIC STRAIN WTHOUT CABLES

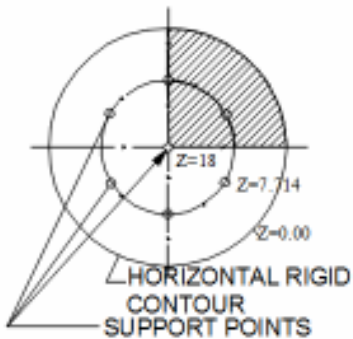

Y 4 HATCHED AREA DIGITIZATION

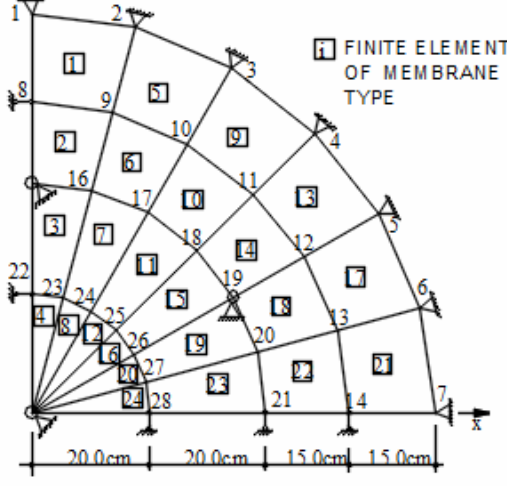

LINE 1-15-29
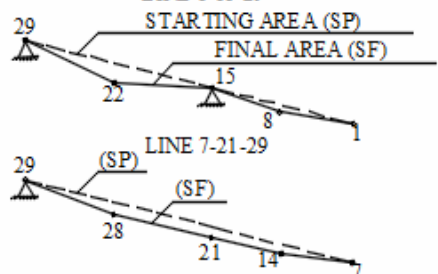

NUMBER OF UNKNOWN: 52

Figure 8. Shape for circular hall with membrane. Mechanical stress

Material: Soap film with stress $\mathrm{T}=1,0 \mathrm{daN} / \mathrm{cm}$

\begin{tabular}{|c|c|c|c|}
\hline \multirow{2}{*}{ Point } & \multicolumn{3}{|c|}{ Coordinates after 8 iterations [cm] } \\
\cline { 2 - 4 } & $\mathrm{x}$ & $\mathrm{y}$ & $\mathrm{z}$ \\
\hline 8. & 0 & 54,390 & 2,731 \\
\hline 9. & 14,109 & 52,824 & 2,739 \\
\hline 10. & 27,306 & 47,293 & 2,579 \\
\hline 11. & 38,688 & 38,615 & 2,742 \\
\hline 12. & 47,102 & 27,195 & 2,730 \\
\hline 13. & 52,797 & 14,209 & 2,739 \\
\hline 14. & 54,611 & 0 & 2,579 \\
\hline 15. & 0 & 40,000 & 7,714 \\
\hline 16. & 10,074 & 38,047 & 5,881 \\
\hline 17. & 19,615 & 33,972 & 5,473 \\
\hline 18. & 27,910 & 27,736 & 5,883 \\
\hline 19. & 34,640 & 20,000 & 7,714 \\
\hline 20. & 37,984 & 10,312 & 5,882 \\
\hline 21. & 39,229 & 0 & 5,472 \\
\hline 22. & 0 & 18,521 & 8,841 \\
\hline 23. & 4,691 & 17,632 & 9,174 \\
\hline 24. & 9,095 & 15,752 & 9,219 \\
\hline
\end{tabular}




\begin{tabular}{|r|c|c|c|}
\hline 25. & 12,923 & 12,873 & 9,175 \\
\hline 26. & 16,039 & 9,260 & 8,841 \\
\hline 27. & 17,614 & 4,759 & 9,174 \\
\hline 28. & 18,190 & 0 & 9,219 \\
\hline 29. & 0 & 0 & 18,000 \\
\hline
\end{tabular}

Table 3. Coordinates after 8 iterations with stress $\mathrm{T}=1,0 \mathrm{daN} / \mathrm{cm}$

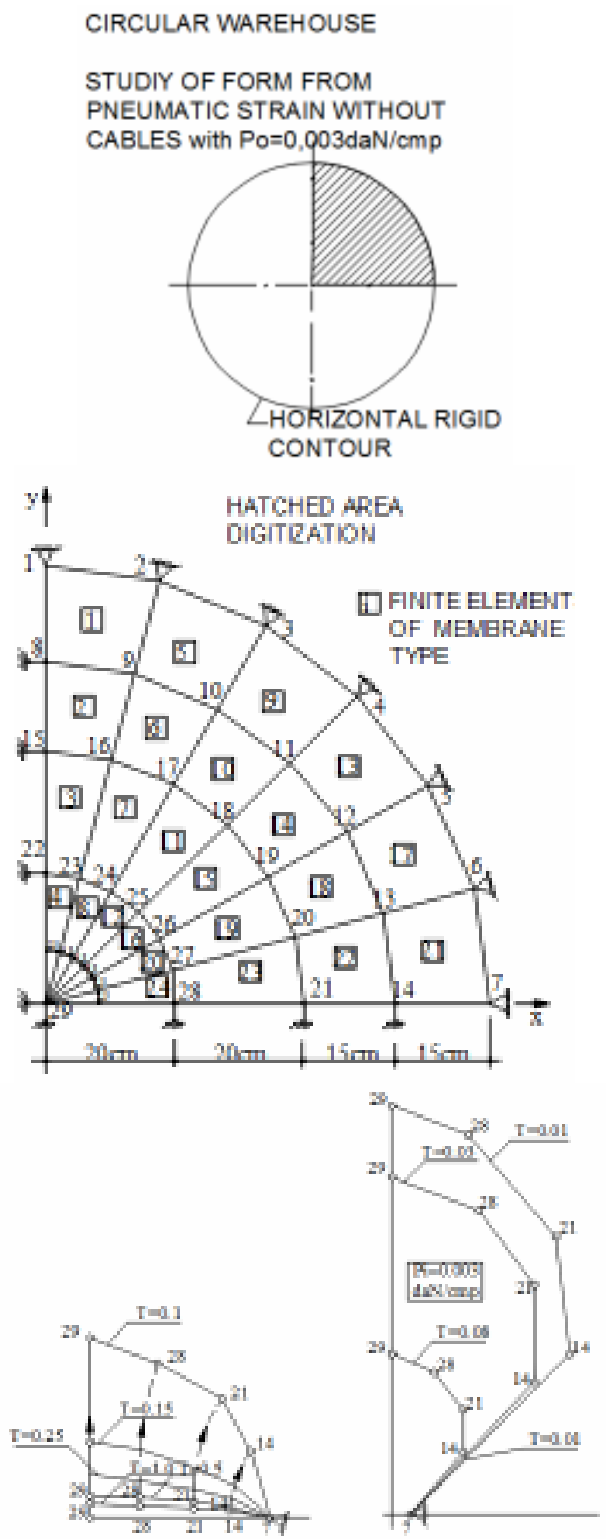

Figure 9. Shape for a circular hall with membrane

\begin{tabular}{|c|c|c|c|}
\hline \multirow{2}{*}{ Point } & \multicolumn{3}{|c|}{ Coordinates after 8 iterations $[\mathrm{cm}]$} \\
\hline & $\mathrm{x}$ & $\mathrm{Y}$ & $\mathrm{Z}$ \\
\hline \multirow{9}{*}{12} & 47,439 & 27,390 & 1,413 \\
\hline & 47,628 & 27,499 & 2,828 \\
\hline & 48,032 & 27,733 & 5,828 \\
\hline & 48,930 & 28,251 & 10,54 \\
\hline & 54,918 & 31,709 & 25,84 \\
\hline & 251,43 & 145,18 & 231,76 \\
\hline & 515,83 & 297,86 & 518,47 \\
\hline & 587,86 & 339,44 & 624,27 \\
\hline & 587,01 & 338,96 & 623,13 \\
\hline \multirow{9}{*}{19} & 34,275 & 19,789 & 2,483 \\
\hline & 34,548 & 19,947 & 4,981 \\
\hline & 35,126 & 20,281 & 10,270 \\
\hline & 36,304 & 20,961 & 19,009 \\
\hline & 43,378 & 25,046 & 44,968 \\
\hline & 245,44 & 141,72 & 413,37 \\
\hline & 497,44 & 287,22 & 904,47 \\
\hline & 551,86 & 318,65 & 1131,01 \\
\hline & 551,54 & 318,46 & 1129,71 \\
\hline \multirow{9}{*}{21} & 39,578 & 0 & 2,483 \\
\hline & 39,894 & 0 & 4,981 \\
\hline & 40,561 & 0 & 10,270 \\
\hline & 41,992 & 0 & 19,009 \\
\hline & 50,089 & 0 & 44,968 \\
\hline & 283,41 & 0 & 413,37 \\
\hline & 574,39 & 0 & 904,48 \\
\hline & 637,23 & 0 & 1131,02 \\
\hline & 636,86 & 0 & 1129,72 \\
\hline \multirow{9}{*}{26} & 16,832 & 9,718 & 3,394 \\
\hline & 17,074 & 9,858 & 6,830 \\
\hline & 17,607 & 10,166 & 14,106 \\
\hline & 18,643 & 10,764 & 25,971 \\
\hline & 23,811 & 13,748 & 60,763 \\
\hline & 151,30 & 87,35 & 560,51 \\
\hline & 295,31 & 170,70 & 1201,39 \\
\hline & 287,44 & 165,96 & 1493,88 \\
\hline & 287,47 & 165,98 & 1493,27 \\
\hline \multirow{9}{*}{28} & 19,437 & 0 & 3,394 \\
\hline & 19,716 & 0 & 6,830 \\
\hline & 20,331 & 0 & 14,106 \\
\hline & 21,528 & 0 & 25,971 \\
\hline & 27,495 & 0 & 60,763 \\
\hline & 174,71 & 0 & 560,51 \\
\hline & 341,01 & 0 & 1201,38 \\
\hline & 331,92 & 0 & 1493,87 \\
\hline & 331,96 & 0 & 1493,2 \\
\hline \multirow{9}{*}{29} & 0 & 0 & 3,765 \\
\hline & 0 & 0 & 7,594 \\
\hline & 0 & 0 & 15,734 \\
\hline & 0 & 0 & 29,028 \\
\hline & 0 & 0 & 68,127 \\
\hline & 0 & 0 & 633,35 \\
\hline & 0 & 0 & 1335,13 \\
\hline & 0 & 0 & 1618,0 \\
\hline & 0 & 0 & 1618 \\
\hline
\end{tabular}

Table 4. Points coordinates $\mathrm{x}, \mathrm{y}, \mathrm{z}[\mathrm{cm}]$ structure. Pneumatic stress 
CIRCULAR WAREHOUSE

STUDY OF FORM FROM

PNEUMATIC STRAIN WITH CABLES

$(\mathrm{Po}=0,003$ daN $/ \mathrm{cmp})$
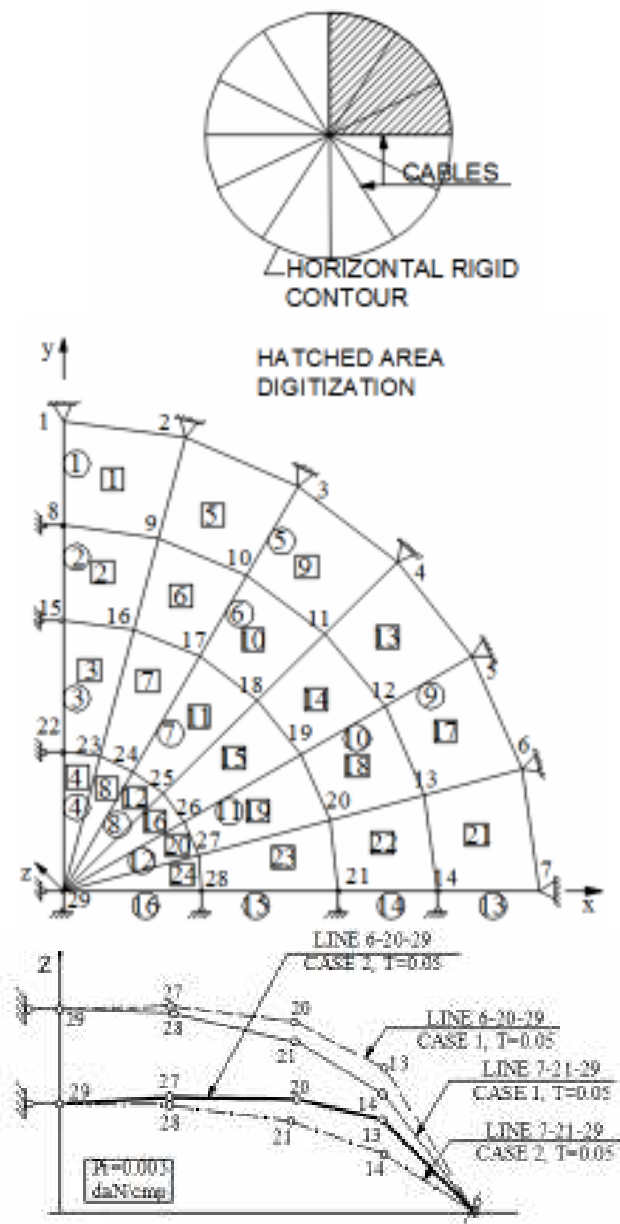

Figure 10. Shape for a circular hall with membrane structure. Pneumatic stress for situation when there are stabilization cables

CASE 1: Initial length $\mathrm{L}_{0}(\mathrm{IJ})_{1}$ of the elements of cable

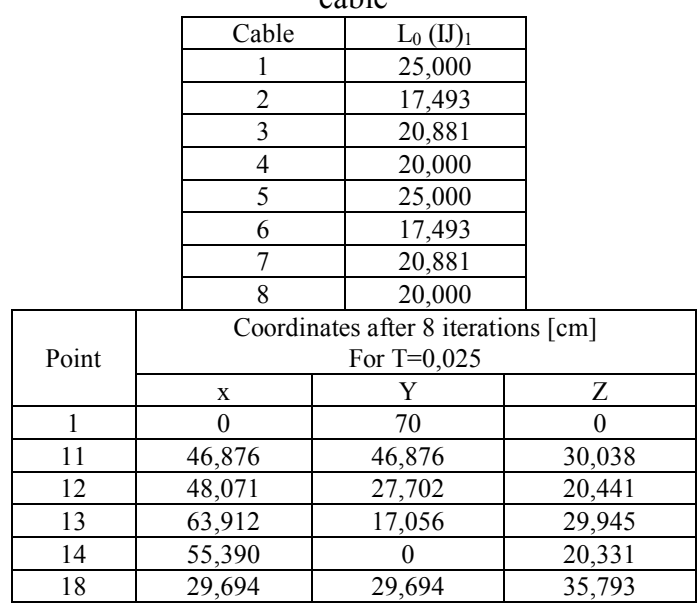

\begin{tabular}{|c|c|c|c|}
\hline 19 & 35,085 & 20,194 & 29,541 \\
\hline 20 & 40,543 & 10,791 & 35,634 \\
\hline 21 & 40,406 & 0 & 29,409 \\
\hline 25 & 15,024 & 15,024 & 35,398 \\
\hline 26 & 17,381 & 9,995 & 34,140 \\
\hline 27 & 20,548 & 5,433 & 35,328 \\
\hline 28 & 20,018 & 0 & 34,064 \\
\hline 29 & 0 & 0 & 34,760 \\
\hline \multirow[t]{2}{*}{ Point } & \multicolumn{3}{|c|}{$\begin{array}{c}\text { Coordinates after } 8 \text { iterations }[\mathrm{cm}] \\
\text { For } \mathrm{T}=0,05\end{array}$} \\
\hline & $\mathrm{x}$ & Y & Z \\
\hline 1 & 0 & 70 & 0 \\
\hline 11 & 39,026 & 39,026 & 24,780 \\
\hline 12 & 47,657 & 27,497 & 20,078 \\
\hline 13 & 53,267 & 14,298 & 24,738 \\
\hline 14 & 54,952 & 0 & 19,996 \\
\hline 18 & 26,971 & 26,971 & 32,296 \\
\hline 19 & 34,880 & 20,115 & 29,539 \\
\hline 20 & 36,818 & 9,865 & 32,242 \\
\hline 21 & 40,210 & 0 & 29,446 \\
\hline 25 & 14,931 & 14,931 & 35,251 \\
\hline 26 & 17,347 & 9,999 & 34,837 \\
\hline 27 & 20,394 & 5,455 & 35,219 \\
\hline 28 & 20,001 & 0 & 34,789 \\
\hline 29 & 0 & 0 & 35,686 \\
\hline \multirow{2}{*}{ Point } & \multicolumn{3}{|c|}{ Coordinates after 8 iterations $[\mathrm{cm}]$ for $\mathrm{T}=0,1$} \\
\hline & $\mathrm{x}$ & $\mathrm{y}$ & $\mathrm{z}$ \\
\hline 1 & 0 & 70 & 0 \\
\hline 11 & 35,807 & 35,807 & 23,029 \\
\hline 12 & 46,825 & 27,036 & 19,286 \\
\hline 13 & 48,919 & 13,122 & 22,999 \\
\hline 14 & 54,048 & 0 & 19,259 \\
\hline 18 & 25,727 & 25,727 & 30,931 \\
\hline 19 & 34,382 & 19,847 & 29,278 \\
\hline 20 & 35,137 & 9,429 & 30,916 \\
\hline 21 & 39,681 & 0 & 29,248 \\
\hline 25 & 14,745 & 14,745 & 35,951 \\
\hline 26 & 17,261 & 9,964 & 36,038 \\
\hline 27 & 20,138 & 5,397 & 35,943 \\
\hline 28 & 19,924 & 0 & 36,024 \\
\hline 29 & 0 & 0 & 37,840 \\
\hline
\end{tabular}

Table 5. Points coordinates: $\mathrm{x}, \mathrm{y}, \mathrm{z}[\mathrm{cm}]$

CASE 2: Initial length $\mathrm{L}_{0}(\mathrm{IJ})_{2}$ of the elements of cable

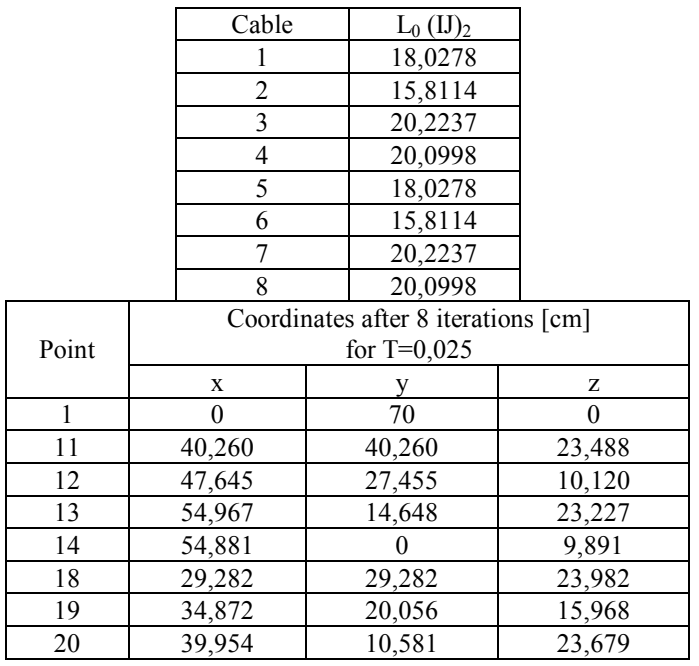




\begin{tabular}{|c|c|c|c|}
\hline 21 & 40,125 & 0 & 19,667 \\
\hline 25 & 19,888 & 19,888 & 20,954 \\
\hline 26 & 17,503 & 10,046 & 19,224 \\
\hline 27 & 27,194 & 7,237 & 20,757 \\
\hline 28 & 20,137 & 0 & 19,032 \\
\hline 29 & 0 & 0 & 19,600 \\
\hline \multirow[t]{2}{*}{ Point } & \multicolumn{3}{|c|}{$\begin{array}{c}\text { Coordinates after } 8 \text { iterations }[\mathrm{cm}] \\
\text { For } \mathrm{T}=0,05\end{array}$} \\
\hline & $\mathrm{x}$ & $\mathrm{y}$ & $\mathrm{z}$ \\
\hline 1 & 0 & 70 & 0 \\
\hline 11 & 34,548 & 34,548 & 17,021 \\
\hline 12 & 47,508 & 27,403 & 9,875 \\
\hline 13 & 47,156 & 12,709 & 16,892 \\
\hline 14 & 54,754 & 0 & 9,682 \\
\hline 18 & 25,422 & 25,422 & 19,456 \\
\hline 19 & 34,794 & 20,048 & 15,873 \\
\hline 20 & 34,701 & 9,341 & 19,322 \\
\hline 21 & 40,071 & 0 & 15,623 \\
\hline 25 & 18,956 & 18,956 & 19,699 \\
\hline 26 & 17,474 & 10,056 & 19,330 \\
\hline 27 & 25,903 & 6,995 & 19,588 \\
\hline 28 & 20,126 & 0 & 19,187 \\
\hline 29 & 0 & 0 & 19,867 \\
\hline \multirow{2}{*}{ Point } & \multicolumn{3}{|c|}{ Coordinates after 8 iterations $[\mathrm{cm}]$ for $\mathrm{T}=0,1$} \\
\hline & $\mathrm{x}$ & $\mathrm{Y}$ & $\bar{Z}$ \\
\hline 1 & 0 & 70 & 0 \\
\hline 11 & 31,984 & 31,984 & 15,042 \\
\hline 12 & 47,292 & 27,310 & 9,463 \\
\hline 13 & 43,753 & 11,735 & 14,941 \\
\hline 14 & 54,556 & 0 & 9,339 \\
\hline 18 & 24,293 & 24,293 & 17,819 \\
\hline 19 & 34,655 & 20,013 & 15,635 \\
\hline 20 & 33,189 & 8,934 & 17,748 \\
\hline 21 & 39,965 & 0 & 15,478 \\
\hline 25 & 18,922 & 18,922 & 19,060 \\
\hline 26 & 17,419 & 10,064 & 19,448 \\
\hline 27 & 25,871 & 6,938 & 19,003 \\
\hline 28 & 20,096 & 0 & 19,371 \\
\hline 29 & 0 & 0 & 20,425 \\
\hline
\end{tabular}

Table 5. Points coordinates: $\mathrm{x}, \mathrm{y}, \mathrm{z}[\mathrm{cm}]$

\section{CONCLUSIONS}

- In order to cover large areas (ths $\mathrm{m}^{2}$ ) membrane type light structures are suitable design options.

- $n$ case of such structures, the shape is the structure and the structure coincides with the shape, so any solution that resolves the optimal and stable shape has a practical importance.

- This paper presents a numerical procedure for finding the shape by using finite elements with constant stress.

\section{REFERENCES}

Bentley, P., 1999, Evolutinary Design by Computers. Morgan Kaufmann, Burlington.

Burry, M., 2011, Scripting Cultures; Architectural Design and Programming. Wiley, London.
Cătărig, A., Kopenetz, L., 1998, Structuri uşoare alcătuite din cabluri şi membrane (Cables and Membranes Structures), Editura UT Pres, Cluj-Napoca.

Fox, M., Kemp, P., 2009, Iteractive Architecture. Princeton Architectural Press, New York.

Hawking, S., Mlodinov, L., 2010, The Grand Design. Banton Books, New York.

Kopenetz, L., Pârv, Bianca, 2014, Introducere în teoria structurilor înalte şi a structurilor cu dimensiuni mari (Introduction into High and Big Dimensions Structures Theory). Editura UT Pres, Cluj-Napoca.

Kopenetz, L., Prada, Marcela, 2011, Introducere în teoria structurilor speciale (Introduction into Special Structures Theory) . Editura Universității, Oradea.

Kopenetz, L., 2006, Gongolatok Statikusoknak. Ed. Kriterion, Cluj-Napoca.

Kopenetz, L., Cătărig, A., Alexa, P., 2004, Setting the Form of Light Membrane Structures. Proceedings of International Conference Performance based Engineering for $21^{s t}$ Century, Iaşi.

Kopenetz, L., Cătărig, A., Alexa, P., 2005, The Geometry of Stressed Lightweight Structures. International Colloquium of IASS Polish Chapter, Warsav.

Kopenetz, L., Cătărig, A., 2006, Teoria structurilor uşoare cu cabluri şi membrane (Membranes and Cables Light Structures Theory), Editura UT Pres, Cluj-Napoca.

Kopenetz, L., Ionescu, A., 1985, Lightweight Roof for Dwellings. International Journal for Housing and its Application, Vol.9, Nr.3, Miami, SUA.

Kwinter, S., 2007, Far from Equilibrium - Essay on Technology and Design Culture. Actar, Barcelona.

Lynn, G., 1999, Animate Form. Princeton Architectural Press, New York.

Plateau, J.A.F., 1873, Statique experimentale et theoretique des liquids, Paris

Rubinstein, M.F., Firstenberg, I.R., 1999, The Minding Organization. Wiley, London.

Woodbury, P., 2010, Elements of Parametric Design. Routledge, London. 